University of Nebraska - Lincoln

DigitalCommons@University of Nebraska - Lincoln

2005

\title{
MALDI-TOF MS Characterization of Carboxyl-End-Capped Polystyrenes Synthesized Using Anionic Polymerization
}

Haining Ji

William K. Nonidez

Rigoberto C. Advincula

Grant D. Smith

S. Michael Kilbey II

See next page for additional authors

Follow this and additional works at: https://digitalcommons.unl.edu/usdoepub

Part of the Bioresource and Agricultural Engineering Commons

Ji, Haining; Nonidez, William K.; Advincula, Rigoberto C.; Smith, Grant D.; Kilbey II, S. Michael; Dadmun, Mark D.; and Mays, Jimmy W., "MALDI-TOF MS Characterization of Carboxyl-End-Capped Polystyrenes Synthesized Using Anionic Polymerization" (2005). US Department of Energy Publications. 59.

https://digitalcommons.unl.edu/usdoepub/59

This Article is brought to you for free and open access by the U.S. Department of Energy at DigitalCommons@University of Nebraska - Lincoln. It has been accepted for inclusion in US Department of Energy Publications by an authorized administrator of DigitalCommons@University of Nebraska - Lincoln. 


\section{Authors}

Haining Ji, William K. Nonidez, Rigoberto C. Advincula, Grant D. Smith, S. Michael Kilbey II, Mark D. Dadmun, and Jimmy W. Mays 


\title{
MALDI-TOF MS Characterization of Carboxyl-End-Capped Polystyrenes Synthesized Using Anionic Polymerization
}

\author{
Haining Ji, ${ }^{\dagger}$ William K. Nonidez, ${ }^{\ddagger}$ Rigoberto C. Advincula, ${ }^{\S}$ Grant D. Smith,, \\ S. Michael Kilbey II, ${ }^{\perp}$ Mark D. Dadmun, ${ }^{\dagger}$ and Jimmy W. Mays ${ }^{*, \dagger, \#}$ \\ Department of Chemistry, University of Tennessee at Knoxville, Knoxville, Tennessee 37996; \\ Department of Chemistry, University of Alabama at Birmingham, Birmingham, Alabama 35294; \\ Department of Chemistry, University of Houston, Houston, Texas 77204; Department of Materials \\ Science \& Engineering, Department of Chemical \& Fuel Engineering, University of Utah, \\ Salt Lake City, Utah 84112; Department of Chemical Engineering, Clemson University, \\ Clemson, South Carolina 29634; and Chemical Sciences Division, Oak Ridge National Laboratory,
}

Oak Ridge, Tennessee 37831

Received April 18, 2005; Revised Manuscript Received September 21, 2005

\begin{abstract}
A comparative study of three strategies used to synthesize well-defined carboxyl-end-capped polystyrenes is reported. Structural characterization was carried out using a combination of thin-layer chromatography (TLC) and matrix-assisted laser desorption/ionization time-of-flight mass spectrometry (MALDI-TOF MS). For the reaction of polystyryl anions with 4-bromo-1,1,1-trimethoxybutane, in addition to the major product, $\mathrm{Bu}-\mathrm{PS}-\mathrm{CH}_{2} \mathrm{CH}_{2} \mathrm{CH}_{2} \mathrm{COOH}$, four byproducts were observed. Their mass values suggest the following structures: $\mathrm{Bu}-\mathrm{PS}-\mathrm{H}, \mathrm{Bu}-\mathrm{PS}-\mathrm{CH}_{3}, \mathrm{Bu}-\mathrm{PS}-\mathrm{CH}_{2} \mathrm{CH}=\mathrm{CH}-\mathrm{CHO}$, and $\mathrm{Bu}-\mathrm{PS}-$ $\mathrm{CH}_{2} \mathrm{CH}_{2} \mathrm{CH}_{2}-\mathrm{CO}-\mathrm{PS}-\mathrm{Bu}$. The reaction of polystyryl anions with succinic anhydride generated the targeted compound, $\mathrm{Bu}-\mathrm{PS}-\mathrm{CO}-\mathrm{CH}_{2} \mathrm{CH}_{2} \mathrm{COOH}$, but also yielded the byproducts $\mathrm{Bu}-\mathrm{PS}-\mathrm{H}$ and dimer $\mathrm{Bu}-\mathrm{PS}-\mathrm{CO}-\mathrm{CH}_{2} \mathrm{CH}_{2}-\mathrm{CO}-\mathrm{PS}-\mathrm{Bu}$. Under appropriate conditions, the reaction of carbon dioxide gas with polystyryl anions could yield quantitative carboxyl chain-end-functionalization.
\end{abstract}

\section{Introduction}

Anionic polymerization is the most powerful method for making end-functionalized polymers since it provides polymer backbones of predictable molecular weights and narrow molecular weight distributions, and the longlived anionic chain-ends readily react with suitable reagents to form functional end groups in high yield. ${ }^{1-3}$ Strategies for synthesizing end-functionalized polymers generally fall into three categories. Some compounds can react directly with the living polymeric anions, thus generating the desired end-functionalized polymer. For example, well-defined hydroxyl-terminated and sulfonate-terminated polymers can be obtained by treating the anionic living polymers with slight excess amounts of ethylene oxide and 1,3-propane sultone, respectively. ${ }^{4,5}$ A second synthetic strategy to end-functionalized polymers involves treating the polymeric anions with alkyl halides (RX), where $\mathrm{R}$ represents alkyl groups containing protective moieties. ${ }^{6-8}$ Suitable protective moieties that are stable toward the anionic living polymers and can be later converted to the desired functional end groups are necessary, and these reactions are generally best carried out in polar solvents at low temperature to minimize side reactions. A third strategy involves adding the living anionic polymer into a large excess of a reagent that contains two of the desired functional groups, which can also react directly with the polyanions. Through control of stoichiometry the formation of dimer may be minimized. Figure 1 illustrates these three general methods for synthesizing carboxyl-

\footnotetext{
$\dagger$ University of Tennessee at Knoxville.

$\ddagger$ University of Alabama at Birmingham.

$\S$ University of Houston.

" University of Utah.

${ }^{\perp}$ Clemson University

\# Oak Ridge National Laboratory.

* Corresponding author.
}

end-capped polystyrene. The first method, reported by Quirk and co-workers, ${ }^{9}$ involved the introduction of carbon dioxide gas $\left(\mathrm{CO}_{2}\right)$ into solutions of living polystyryllithium containing Lewis bases, such as tetrahydrofuran (THF), producing well-defined carboxylend-capped polystyrene in high yield. These same authors ${ }^{9}$ further demonstrated that the reaction of gaseous $\mathrm{CO}_{2}$ with freeze-dried living polystyryllithium in the presence of $N, N, N^{\prime}, N^{\prime}$-tetramethylethylenediamine (TMEDA) resulted in quantitative carboxylation. Hirao and co-workers reported another carboxylation method, 10 belonging to the second category described above, in which living polystyryl anions were reacted with 4-bromo1,1,1-trimethoxybutane in $\mathrm{THF}$ at $-78^{\circ} \mathrm{C}$. The resulting polymer was sequentially treated with acid and base. The acid treatment converted the carboxyl-protective moiety, the ortho ester group, into a methyl ester group. The base treatment hydrolyzed this ester, yielding a carboxyl group. This method was reported to result in high yields of carboxylation (>95\%). ${ }^{10} \mathrm{~A}$ third carboxylation method, reported by Hall and co-workers, ${ }^{11}$ involved the reaction of living polyanions with a large excess of succinic anhydride. In the case of poly(tertbutyl acrylate), they reported $>95 \%$ conversion to the carboxylic acid. ${ }^{11}$

End-group identification and determination of the structures of these materials has long been a great challenge for conventional analytical techniques (NMR, IR, titration, etc.). This is because the signals from the end groups are very weak as compared to those from the polymer backbone due to their low concentrations. End-group signals are often indistinguishable from the background noise and thus cannot be quantified reliably. Detection of the end-group signals from byproducts that are present in even smaller amounts is often not achievable with conventional analytical techniques. For this reason, side reactions and byproducts that occurred 
Category \#1:
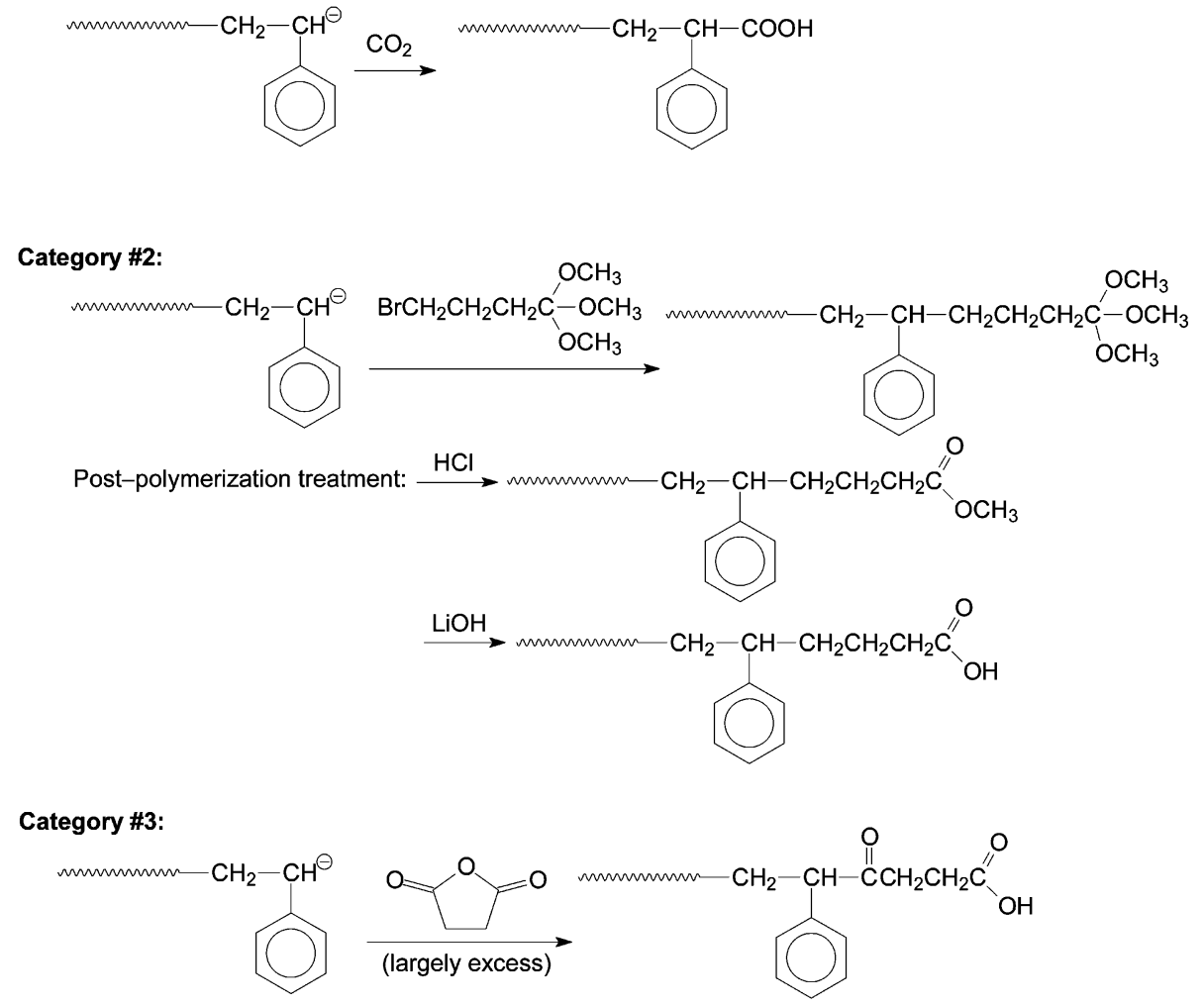

Figure 1. Synthetic strategies to well-defined carboxyl-end-capped polystyrene representing the three categories.

in end-functionalization reactions have seldom been reported in the past.

We are interested in creating and characterizing welldefined telechelic end-functionalized polymers for use in modifying interfaces with doubly bound polymer chains. Recent results have indicated that tethered polymer loops significantly improve the adhesive properties of an interface relative to that of a singly endtethered polymer chain. ${ }^{12-15}$ Interpenetration between two surfaces covered with loops should also be less than that of two polymer brushes. Therefore, the dynamic response of such surfaces should be further enhanced. ${ }^{16-19}$ Thus, the creation of a surface covered with loops should exhibit improved properties compared to traditional singly bound polymer chains in many circumstances. This potential for improvement of properties of polymer-modified surfaces has led us to investigate the ability to reproducibly and controllably produce surfaces that are covered with molecular loops by binding $\alpha, \omega$ telechelic polymers to functionalized surfaces. The synthesis and characterization of the $\alpha, \omega$ telechelic polymers described in this paper is the first step in this project.

The advent of matrix-assisted laser desorption/ionization time-of-flight mass spectrometry (MALDI-TOF MS) provides the possibility for accurately determining the structures of macromolecules. ${ }^{20,21}$ Application of MALDITOF MS to examine the products arising from the preparation of end-functionalized polymer by living anionic polymerization techniques has provoked intense interest during the past 10 years. ${ }^{22-24}$ In some cases, separating such samples according to differences in chemical structure prior to MALDI-TOF MS analysis can greatly simplify the interpretation of the mass spectrum. A number of preseparation techniques in sample preparation for MALDI-TOF MS have been proposed. Examples include size exclusion chromatog- raphy (SEC), ${ }^{25,26}$ high-performance liquid chromatography (HPLC), ${ }^{27,28}$ and thin-layer chromatography (TLC). ${ }^{29,30}$ We have previously reported the use of combined silica gel $\left(\mathrm{SiO}_{2}\right)$-based TLC and MALDI-TOF MS to examine the efficiency of, and side reactions in, some anionic end-functionalization reactions. ${ }^{31,32}$ TLC separates polystyrenes on the basis of the structure of the end group without being much affected by the degree of polymerization. MALDI-TOF mass spectral analysis of the TLC fractions gives information on their structures. This TLC/MALDI-TOF MS method can provide a wealth of information on the reaction products, which yields insight into the reaction mechanisms, and hence the synthesis can be optimized.

In the present work, we reexamine the three reactions shown in Figure 1 using the TLC/MALDI-TOF MS method; to our knowledge this is the first systematic MALDI-TOF MS study of these carboxylation reactions. All end-functionalization reactions in this experiment will be conducted using identical polymer chains, which were synthesized in one batch and then divided into aliquots for the various reactions. Thus, the MALDITOF mass spectra of the resulting products should only reflect differences in their end groups.

\section{Experimental Section}

Materials and Synthetic Manipulations. Polystyryllithium was synthesized in benzene using standard all-glass high-vacuum techniques. ${ }^{33,34}$ Solvent and monomer were purchased from Aldrich and purified according to a standard procedure. ${ }^{34}$ sec-Butyllithium was synthesized by the reaction of sec-butyl chloride (Aldrich, 98\%) and lithium metal (Aldrich, $99.99 \%$ ) and was used as the polymerization initiator. ${ }^{34}$ 4-Bromo-1,1,1-trimethoxybutane was purified over $\mathrm{CaH}_{2}$ twice by fractional distillation and stored in ampules equipped with break-seals. Succinic anhydride was purified by vacuumdrying the solid for at least $12 \mathrm{~h}$ and dilution with purified THF by distillation from a reservoir containing liquid $\mathrm{K} / \mathrm{Na}$ 
alloy ( $3: 1$ by weight) through the high-vacuum line, and then the solution was sealed in a glass apparatus equipped with break-seals. The carbon dioxide gas (Aldrich, 99.8+\%) was used as received. The living anionic polystyrene (PS) solution was divided into six portions by flame-sealing constrictions under vacuum. One aliquot was reacted with methanol to create proton-terminated (nonfunctionalized) PS and used as a reference material for the end-functionalized PSs. Two portions were reacted with a slight excess amount of 1,1-diphenylethylene (DPE) to generate (1,1-diphenylalkyl)lithium chain ends. These six portions were used in the following reactions.

Termination Reactions by Carbon Dioxide Gas. Polystyryllithium was reacted with carbon dioxide gas under two different conditions. One portion of the living polystyryllithium solution was freeze-dried, and carbon dioxide gas was introduced through a rubber septum. The fine orange powder turned snow white immediately as the $\mathrm{CO}_{2}$ gas was injected. This product was dissolved into a $1 \% \mathrm{HCl} / \mathrm{THF}(\mathrm{v} / \mathrm{v})$ solution and precipitated in methanol. Another portion of the living polystyryllithium solution $(15 \mathrm{~mL}$, ca. $10 \%$, w/v) was added to $35 \mathrm{~mL}$ of purified THF at $-78{ }^{\circ} \mathrm{C}$. Carbon dioxide gas was injected through a rubber septum while the solution was stirred vigorously. The color of the living polymer disappeared rapidly. A volume of $0.5 \mathrm{~mL}$ of concentrated $\mathrm{HCl}$ was added to this solution, and the polymer was purified by repeated precipitation from THF into methanol.

Termination Reactions Using 4-Bromo-1,1,1-trimethoxybutane. A portion of polystyryllithium was reacted with 4-bromo-1,1,1-trimethoxybutane in a $\mathrm{THF} / \mathrm{benzene}$ mixture $(7 / 3, \mathrm{v} / \mathrm{v})$ at $-78{ }^{\circ} \mathrm{C}$ for $2 \mathrm{~h}$ using a $[\mathrm{Br}] /[\mathrm{Li}]$ ratio of 4 . This involved addition of 4-bromo-1,1,1-trimethoxybutane into the living polymer solution in THF. Within a few seconds, the characteristic orange-red color of the polystyryl anions was observed to disappear completely, indicating the reaction takes place rapidly. We let the reaction proceed for $2 \mathrm{~h}$ and then terminated it by adding an aliquot of degassed methanol. The reaction mixture was poured into a large excess of methanol to collect the polymer. This polymer was then reprecipitated from THF into methanol. Deprotection was accomplished by sequential treatment of the polymer with $0.1 \mathrm{~N} \mathrm{HCl}$ and $0.2 \mathrm{~N} \mathrm{LiOH}$ solution in THF. Polymer (1.5 g, $\sim 0.4$ mmol) was dissolved in $36 \mathrm{~mL}$ of THF, $4 \mathrm{~mL}$ of $1 \mathrm{~N} \mathrm{HCl}$ was added, and this polymer solution was stirred at room temperature for $24 \mathrm{~h}$. The polymer was precipitated in excess methanol. Then $1.5 \mathrm{~g}$ of polymer was dissolved in $20 \mathrm{~mL}$ of THF. A $0.2 \mathrm{~N} \mathrm{LiOH}$ aqueous solution was used to neutralize this solution. Then $0.096 \mathrm{~g}$ of $\mathrm{LiOH}$ was added, and the solution was stirred under reflux for $24 \mathrm{~h}$. The acid treatment converted the ortho ester groups into methyl ester groups, and the base treatment hydrolyzed the methyl ester groups yielding carboxyl-end-capped polystyrene. A portion of the polymer that was end-capped with DPE was also treated in the same manner.

Termination Reactions Involving Succinic Anhydride. A portion of polystyryllithium was reacted with succinic anhydride in a THF/benzene mixture $\left(7 / 3\right.$, v/v) at $-78^{\circ} \mathrm{C}$ for $2 \mathrm{~h}$. A THF solution of the anionic living polymer was prepared as described above. Succinic anhydride solution was made $\sim 2$ $\mathrm{M}$ in THF. A volume of $10 \mathrm{~mL}$ of succinic anhydride solution was added rapidly to the THF solution of the living polymer at $-78{ }^{\circ} \mathrm{C}$ through the rupture of a break-seal. The solution turned light yellow immediately upon the mixing. This reaction was also allowed to proceed for $2 \mathrm{~h}$, at which time the light yellow color persisted. The solution turned colorless when it was exposed to air. A volume of $0.5 \mathrm{~mL}$ of concentrated hydrochloric acid was added into the reaction mixture. This polymer was purified by repeat precipitation from THF into methanol. A portion of the polymer that was end-capped with DPE was also treated in the same manner.

TLC/MALDI-TOF MS Analysis of the Products. All MALDI-TOF MS measurements were done on a Voyager DE Pro MALDI-TOF MS unit from PE PerSeptive Biosystems (Framingham, MA). The instrument is equipped with a $\mathrm{N}_{2}$ laser $(\lambda=337 \mathrm{~nm})$ with a pulse width of $3 \mathrm{~ns}$, a $2 \mathrm{GHz}$ acquisition digitizer, and a pulsed ion extraction source.
trans,trans-1,4-Diphenyl-1,3-butadiene was used as the matrix in all MALDI-TOF MS measurements. The reason we chose this matrix is threefold: (1) this compound has been reported to be a very efficient matrix material for use with a $337 \mathrm{~nm}$ laser, ${ }^{35}$ (2) its structure is similar to the repeating unit of polystyrene which makes it more likely to form homogeneous cocrystals with our polymers and hence generate mass spectra of good quality, and (3) it is a nonionic compound so it will not complicate the ionization of the samples. Ionization of synthetic polymers usually requires cationization by an adduct of salt rather than protonation. For unsaturated polymer such as PS, transition ions such as $\mathrm{Ag}^{+}$and $\mathrm{Cu}^{+}$are commonly used. ${ }^{36}$ Silver trifluoroacetate (AgTFA) and copper(I) chloride $\left(\mathrm{Cu}{ }^{\mathrm{I} C l}\right)$ were used as the cationizing reagent when the measurement was carried out in positive-ion mode. All positive-ion spectra displayed in this paper were caused by the addition of $\mathrm{Ag}^{+}$. Copper(I) cationization reagent was used to confirm that the multiple distributions appearing in one mass spectrum were caused by multiple compounds rather than different ion additions. All negative-ion spectra were obtained without any ionizing agents added.

TLC plates $(200 \mu \mathrm{m}$, with fluorescent indicator F-254), purchased from Scientific Absorbents (Atlanta, GA), were employed to purify the products for MALDI-TOF MS analysis. Toluene was used as the developing solvent in all TLC separations. The spots of polymers were scraped from the developed TLC plate along with the silica gel. A volume of 50 $\mu \mathrm{L}$ of THF was added to each sample to extract the polymers. A volume of $20 \mu \mathrm{L}$ of the polymer solution was mixed with 20 $\mu \mathrm{L}$ of the matrix solution ( $0.1 \mathrm{M}$ in THF). For each spectrum that was acquired in positive-ion mode, a volume of $1 \mu \mathrm{L}$ of the THF solution of AgTFA ( $5 \mathrm{mg} / \mathrm{mL}$ ) was added to the polymer/matrix mixture. The dried droplet method ${ }^{37}$ was used to prepare the sample target: $1 \mu \mathrm{L}$ of the polymer/matrix/ cation mixture solution was applied to the sample target, and the solvent was evaporated by air-drying. In the case of spectra acquired in negative-ion mode, no additional ion was added. The polymer/matrix mixture was applied to the sample target by the dried droplet method.

All mass spectra were obtained in linear mode. The accelerating voltage was $25 \mathrm{kV}$. Each spectrum displayed in this article is the average of 128 single-shot spectra. The mass scale in the linear mode was calibrated externally using the peptides: angiotensin-I $(M=1296.5)$ and insulin bovine pancreas ( $M=5733.6$ ) (Sigma, St. Louis, MO) as standards. $\mathrm{M}+\mathrm{H}$ values were used to calibrate the positive-ion mode, and $\mathrm{M}-\mathrm{H}$ values were used to calibrate the negative-ion mode.

Size Exclusion Chromatography (SEC). SEC was used to determine the average molecular weights and molecular weight distributions, $M_{\mathrm{w}} / M_{\mathrm{n}}$, of the polymer samples with respect to polystyrene standards (Polymer Standards Service (PPS), Germany). Measurements were done on a Polymer Labs GPC-120 unit equipped with an isocratic pump (Agilent 1100 series), a Polymer Lab differential refractive index detector, and two Polymer Labs PLgel $10 \mu \mathrm{m}$ MIXED-B columns (pore size: $8-10 \mu \mathrm{m}$; linear MW range: 500-10 million). Data acquisition employed the PL DataStream external interface, and data were analyzed using the PL Cirrus (version 1.1) software. All samples were run using THF as the mobile phase at a flow rate of $1 \mathrm{~mL} / \mathrm{min}$ at $40^{\circ} \mathrm{C}$.

\section{Results and Discussion}

The crude product from the reaction of living polystyryl anions with purified 4-bromo-1,1,1-trimethoxybutane gives three spots after TLC development. The retardation factor $\left(R_{f}\right)$ values of these spots are (A) 0.95, (B) 0.64 , and (C) 0 . Reaction of living polymeric diphenyl anions with purified 4-bromo-1,1,1-trimethoxybutane gives four spots after TLC development. The $R_{f}$ values of these spots are (D) 0.95, (E) 0.85, (F) 0.64, and (G) 0 . Figure 2 illustrates a TLC image of sample 1 (product from the reaction of polystyryllithium and 4-bromo1,1,1-trimethoxybutane) and sample 2 (product from the 


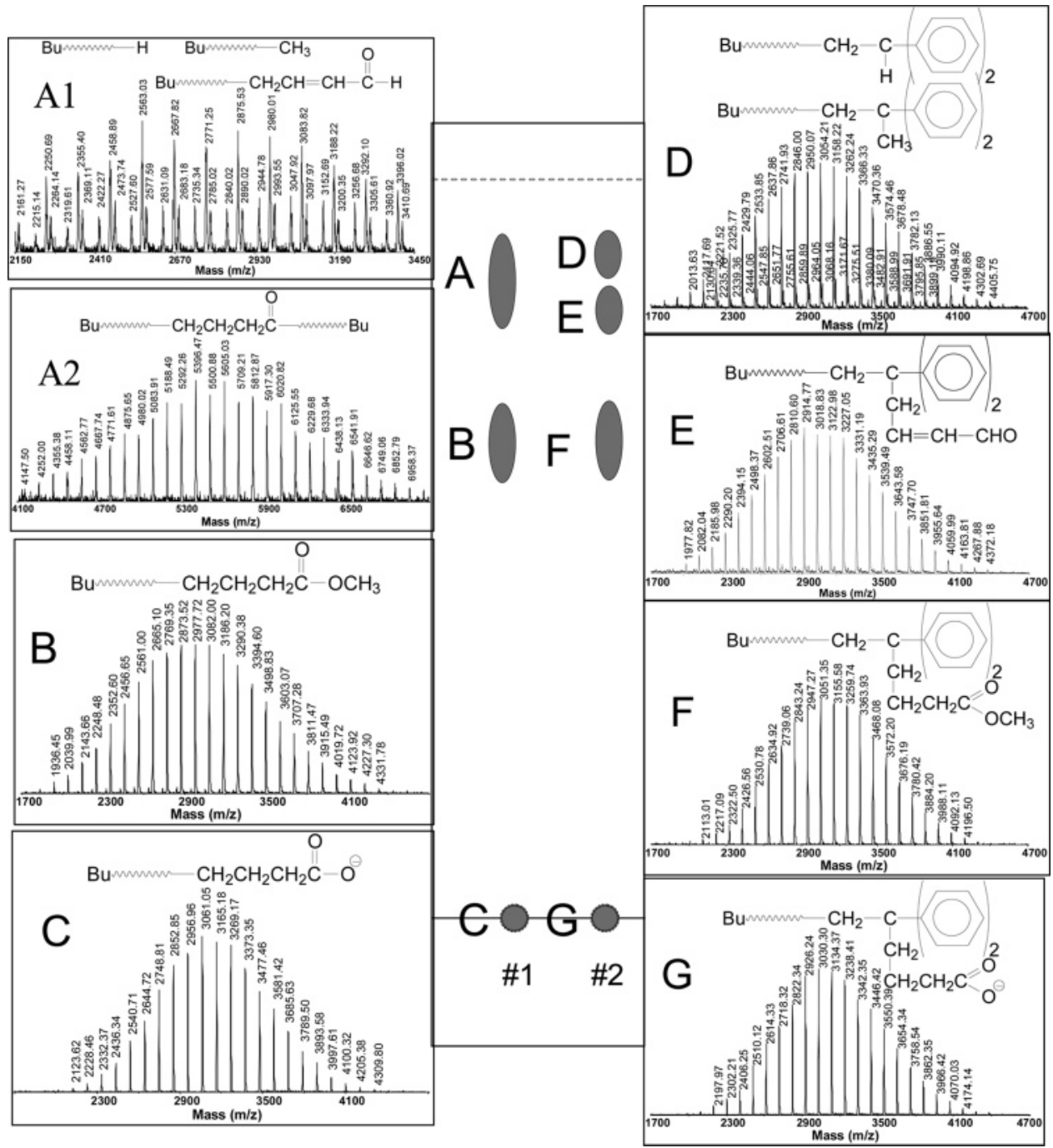

Figure 2. Thin-layer chromatography image of product from (\#1: reaction of polystyryllithium and 4-bromo-1,1,1-trimethoxybutane; \#2: reaction of polymeric (1,1-diphenylalkyl)lithium and 4-bromo-1,1,1-trimethoxybutane) along with the matrixassisted laser desorption/ionization time-of-flight mass spectra of the corresponding spots. Spots A, B, D, E, and F were obtained in positive-ion mode, and the cationization reagent is silver trifluoroacetate. Spots $\mathrm{C}$ and $\mathrm{G}$ were obtained in negative-ion mode. No extra ion was added to these two samples.

reaction of polymeric diphenylalkyllithium and 4-bromo1,1,1-trimethoxybutane) along with the MALDI-TOF mass spectra obtained from the spots on this TLC plate. The mobile phase is toluene, and the stationary phase is silica gel. Under these conditions, polymers having more polar functional groups move slower because they have stronger interactions with the stationary phase. The high mobility of spots A and D indicates the lack of polar functional groups on the polymers in these spots. Likewise, polymer in spots B, E, and F contain functional groups that interact with the stationary phase moderately, and polymer in spots $\mathrm{C}$ and $\mathrm{G}$ contain functional groups that have a strong interaction with the stationary phase.

Spots A, B, D, E, and F give strong signals in the positive-ion mode with the addition of silver cations, and spots $\mathrm{C}$ and $\mathrm{G}$ give strong signals in the negative-ion mode (without addition of ionizing agents) in the MALDI-TOF MS measurements. The mass spectrum of spot A contains not only peaks with the expected molecular weights but also peaks of twice their molecular weights (dimers). A1 shows the low mass part of the mass spectrum of spot A, and A2 shows the high mass part. Multiple distributions are observed in mass spectrum A1. These multiple distributions can be caused by two effects: (1) different specific compounds plus the same cation or (2) single compounds with different cations added (possibly due to contact during synthesis with other cationizing agents). This sample was thus subjected to MALDI-TOF MS analysis using a different cationization reagent (copper(I) chloride). It was found that this caused all peaks to shift 44 mass units lower than the corresponding peaks displayed in mass spectrum A1. This is a strong indication that all peaks in $\mathbf{A 1}$ are caused by individual compounds plus the silver cation.

Two distributions of peaks were also found in mass spectrum $\mathbf{D}$. The molecular weight of each peak in these two distributions matches one of the following structures: (1) $\mathrm{Bu}-\mathrm{PS}-\mathrm{DPE}-\mathrm{H}$ and (2) $\mathrm{Bu}-\mathrm{PS}-\mathrm{DPE}-\mathrm{CH}_{3}$. Here $\mathrm{Bu}$ represents the initiator moiety, a butyl group. For example, the peak that has a molecular weight of 3054.21 is caused by the polymer chains containing 26 repeating units of styrene, a butyl group $(M=57.12)$ at one end, and a DPE unit $(M=180.16)$ and a proton $(M=1.01)$ at the other end. A silver ion $(M=107.87)$ is attached to each of these polymer chains $(26 \times 104.2$ $+57.12+1.01+107.87+180.16=3055.36, \Delta=1.14)$. 


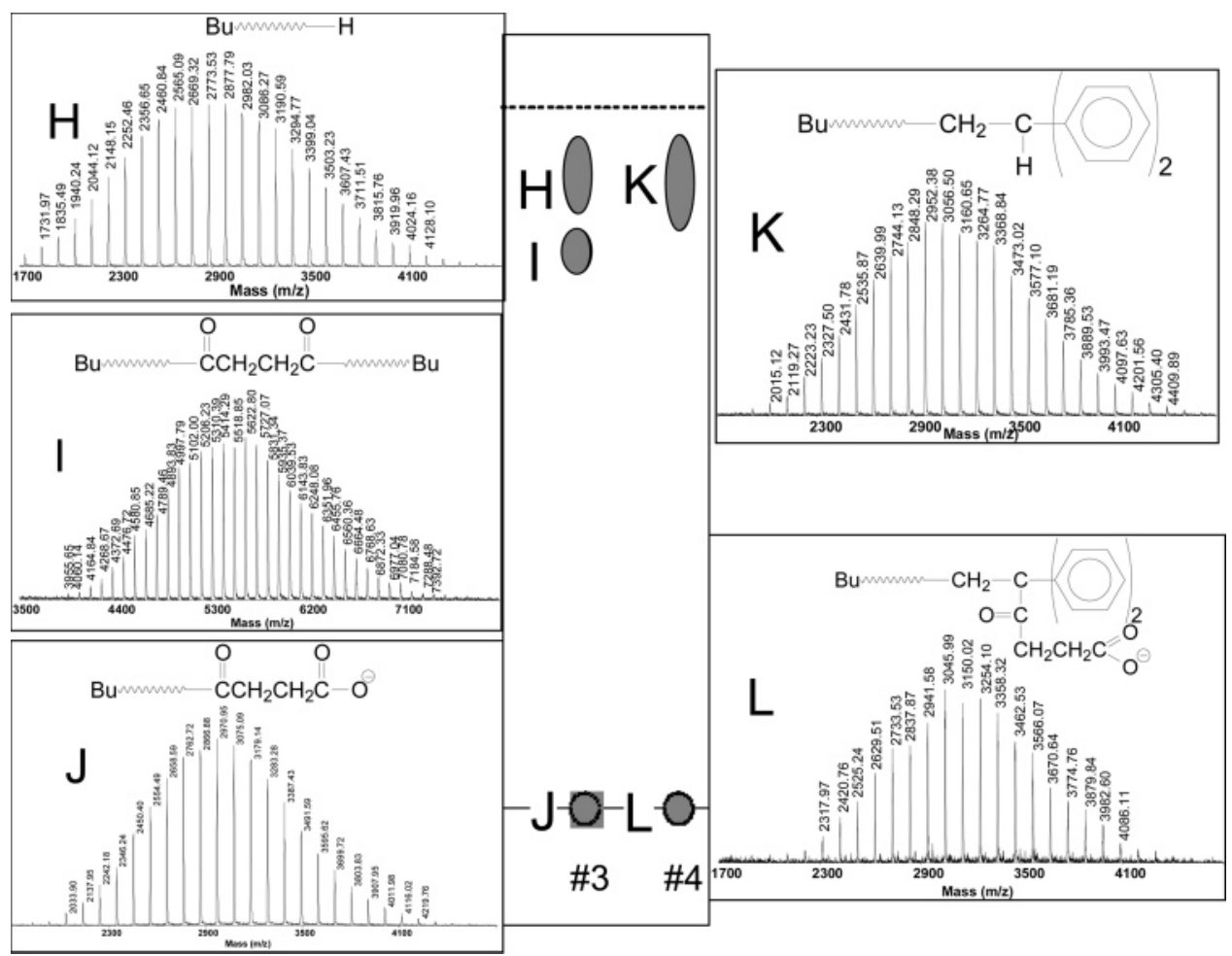

Figure 3. Thin-layer chromatography image of product from (\#3: reaction of polystyryllithium and succinic anhydride; \#4: reaction of polymeric (1,1-diphenylalkyl)lithium and succinic anhydride) along with the matrix-assisted laser desorption/ionization timeof-flight mass spectra of the corresponding spots. Spots H, I, and K were obtained in positive-ion mode, and the cationization reagent is silver trifluoroacetate. Spot $J$ and $L$ were obtained in negative-ion mode. No extra ion was added to these two samples.

The peak that has a molecular weight of 3068.16 is caused by the polymer chains containing 26 repeating units of styrene, a butyl group at one end, and a DPE unit and a methyl group $(M=15.02)$ at the other end. In mass spectrum $\mathbf{E}$, the peak that has a molecular weight of 3122.98 is caused by the polymer chains containing 26 repeating units of styrene, a butyl group at one end, and a DPE unit and a $-\mathrm{CH}_{2}-\mathrm{CH}=\mathrm{C}-\mathrm{CHO}$ group $(M=69.12)$ at the other end. No evidence of dimer is found in sample 2 from the SEC and MALDI-TOF MS measurements.

Mass spectrum A1 contains three distributions of peaks. The molecular weight values of the peaks in these three distributions match the following structures: (1) $\mathrm{Bu}-\mathrm{PS}-\mathrm{H} ;$ (2) $\mathrm{Bu}-\mathrm{PS}-\mathrm{CH}_{3}$, and (3) $\mathrm{Bu}-\mathrm{PS}-\mathrm{CH}_{2} \mathrm{CH}=\mathrm{CH}-\mathrm{CHO}$. The peaks in mass spectrum $\mathrm{A} 2$ match the structure $\mathrm{Bu}-\mathrm{PS}-\mathrm{CH}_{2} \mathrm{CH}_{2} \mathrm{CH}_{2} \mathrm{CO}-$ $\mathrm{PS}-\mathrm{Bu}$. For example, the peak that has a molecular weight of 5605.03 is caused by the polymer chains contain 51 repeating units of styrene plus two butyl group, a silver cation, and the mass of moiety $-\mathrm{CH}_{2} \mathrm{CH}_{2} \mathrm{CH}_{2} \mathrm{CO}-(M=70)$. The mobility of these dimer chains should be lower than (1) and (2) because they have one carbonyl group per chain but higher than (3) since they have longer hydrocarbon chains. The presence of dimer is probably the reason that structures (1), (2), and (3) are mixed in one spot. In the case of sample 2, no dimer was formed from the reaction, and spot E is clearly separated from spot D.

Spots $\mathrm{B}$ and $\mathrm{F}$ have the same mobility $\left(R_{f}=0.64\right)$ in TLC development, and their mass spectra indicate that all chains contained in these two spots have an identical end group $\left(-\mathrm{CH}_{2} \mathrm{CH}_{2} \mathrm{CH}_{2} \mathrm{COOCH}_{3}\right)$. The fact that these two spots disappear after the base treatment further confirms this structural assignment. Spots $\mathrm{C}$ and $\mathrm{G}\left(R_{f}\right.$ $=0$ ) have the least mobility. The peaks in these two spots can be accounted for by the presence of $\mathrm{Bu}-\mathrm{PS}-$ $\mathrm{CH}_{2} \mathrm{CH}_{2} \mathrm{CH}_{2} \mathrm{COO}^{-}$and $\mathrm{Bu}-\mathrm{PS}-\mathrm{CH}_{2} \mathrm{CH}_{2} \mathrm{CH}_{2} \mathrm{CH}_{2} \mathrm{C}(\mathrm{Ph})_{2}-$ $\mathrm{COO}^{-}$, respectively.

Figure 3 displays the TLC image of sample 3 (product from the reaction of polystyryllithium and purified succinic anhydride) and sample 4 (product from the reaction of polymeric diphenylalkyllithium and purified succinic anhydride) along with the MALDI-TOF mass spectra of the corresponding spots. Three spots (H, I, and $J)$ arose from sample 3 after TLC development. The peaks in spot $\mathrm{H}\left(R_{f}=0.95\right)$ are identical to that of the proton-terminated PS reference. The peaks in spot I $\left(R_{f}\right.$ $=0.75$ ) reflect dimers caused by two living polymer chains reacting with the same succinic anhydride molecule, and they are consistent with $\mathrm{Bu}-\mathrm{PS}-\mathrm{CO}-$ $\mathrm{CH}_{2} \mathrm{CH}_{2}-\mathrm{CO}-\mathrm{PS}-\mathrm{Bu}$. The mass spectrum of spot $\mathrm{J}\left(R_{f}\right.$ $=0$ ) was obtained in negative-ion mode. Peaks in this spectrum are consistent with $\mathrm{Bu}-\mathrm{PS}-\mathrm{CO}-\mathrm{CH}_{2} \mathrm{CH}_{2}-$ COO-. Two spots were developed from sample 4. Peaks in spot $\mathrm{K}\left(R_{f}=0.95\right)$ are accounted for $\mathrm{Bu}-\mathrm{PS}-\mathrm{CH}_{2} \mathrm{C}$ $(\mathrm{Ph})_{2}-\mathrm{H}$, and those in spot $\mathrm{L}\left(R_{f}=0\right)$ are $\mathrm{Bu}-\mathrm{PS}-$ $\mathrm{CH}_{2} \mathrm{C}(\mathrm{Ph})_{2}-\mathrm{CO}-\mathrm{CH}_{2} \mathrm{CH}_{2}-\mathrm{COO}-$. No dimer was detected in sample 4.

The TLC image of product from the reaction of polystyryllithium and carbon dioxide gas in the freezedried state and the MALDI-TOF mass spectra of the corresponding spots are displayed in Figure 4. Spot $\mathrm{P}$ $\left(R_{f}=0.95\right)$ contains $\mathrm{Bu}-\mathrm{PS}-\mathrm{H}$ and dimer $\mathrm{Bu}-\mathrm{PS}-$ $\mathrm{PS}-\mathrm{Bu}$. The structures of the dimer peaks indicate that they were caused by oxygen gas instead of carbon dioxide gas. These two byproducts suggest that small amounts of moisture and oxygen also reacted with the living polymers. In the mass spectrum $\mathbf{N}$, all peaks are accounted for $\mathrm{Bu}-\mathrm{PS}-\mathrm{COO}^{-}$. When the reaction of polystyryllithium and carbon dioxide gas was carried out in THF solution at $-78{ }^{\circ} \mathrm{C}$, all chains stay at the 


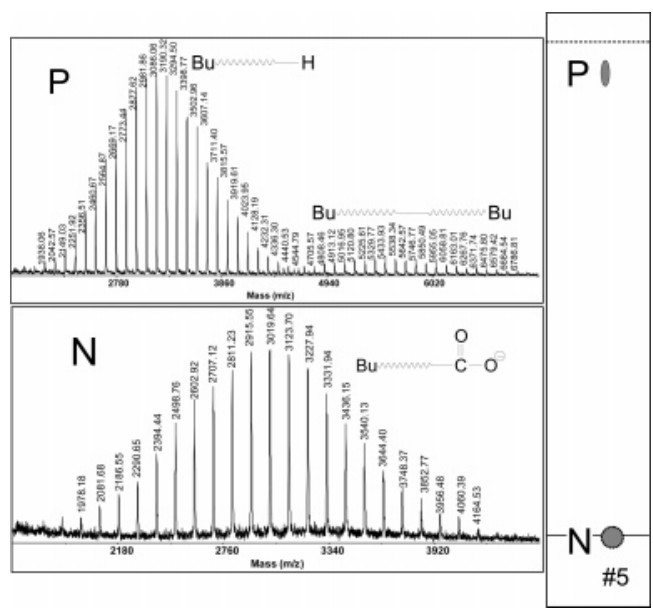

Figure 4. Thin-layer chromatography image of product from the reaction of polystyryllithium and carbon dioxide gas at freeze-dry state along with the matrix-assisted laser desorption/ionization time-of-flight mass spectra of the corresponding spots. Spot $\mathrm{P}$ was obtained in positive-ion mode, and the cationization reagent is silver trifluoroacetate. Spot $\mathrm{N}$ was obtained in negative-ion mode, and no extra ion was added.

origin after TLC development. Its MALDI-TOF mass spectrum is identical to mass spectrum $\mathbf{N}$. No dimer was detected in either SEC or MALDI-TOF MS.

As described above, we have used the offline TLC/ MALDI-TOF MS methodology to determine the structures of the byproducts generated from three reactions that are commonly used to synthesize well-defined carboxyl-end-capped PS. In this manner, byproducts in these reactions were detected and identified that have not been reported previously. It is possible that other byproducts also exist which we could not detect in our study. The reason is twofold. First, there is always a concern with this method involving the potential loss of component(s) during the transfer from the TLC plate to the MALDI target. Using an on-line TLC/MALDITOF MS method can avoid this potential problem; ${ }^{40}$ unfortunately, our laboratory does not have such online measurement capability. Second, spectral suppression, a common phenomenon encountered in desorption techniques, might be responsible for the inability to detect some byproducts. ${ }^{38,39}$

Quantitative characterization of synthetic polymers by MALDI-TOF MS is limited by two major intrinsic problems in the technique: mass discrimination ${ }^{41-44}$ and functional groups dependence. ${ }^{45-47}$ Mass discrimination of samples with high molecular weight polydispersities is attributed to desorption/ionization efficiency and instrumental settings. Generally, larger molecules are more difficult to ionize/desorb and hence produce poorer signals. We have previously demonstrated that the relative ratios of two components in the same sample spot vary with different instrumental settings. ${ }^{48}$ The intensities of the signals from functional groupcontaining samples often do not reflect the true abundances of the molecules due to the interference of the functional groups in the ionization output. For these reasons, the $y$-axis of the MALDI-TOF mass spectra cannot be used in quantitative characterization.

We thus used SEC to determine the extent of dimerization in each synthesis. The SEC chromatograms of products from the four end-functionalization reactions involving polystyryllithium in this study are shown in Figure 5. These curves are analyzed by an iterative curve-fitting procedure in order to determine the extent

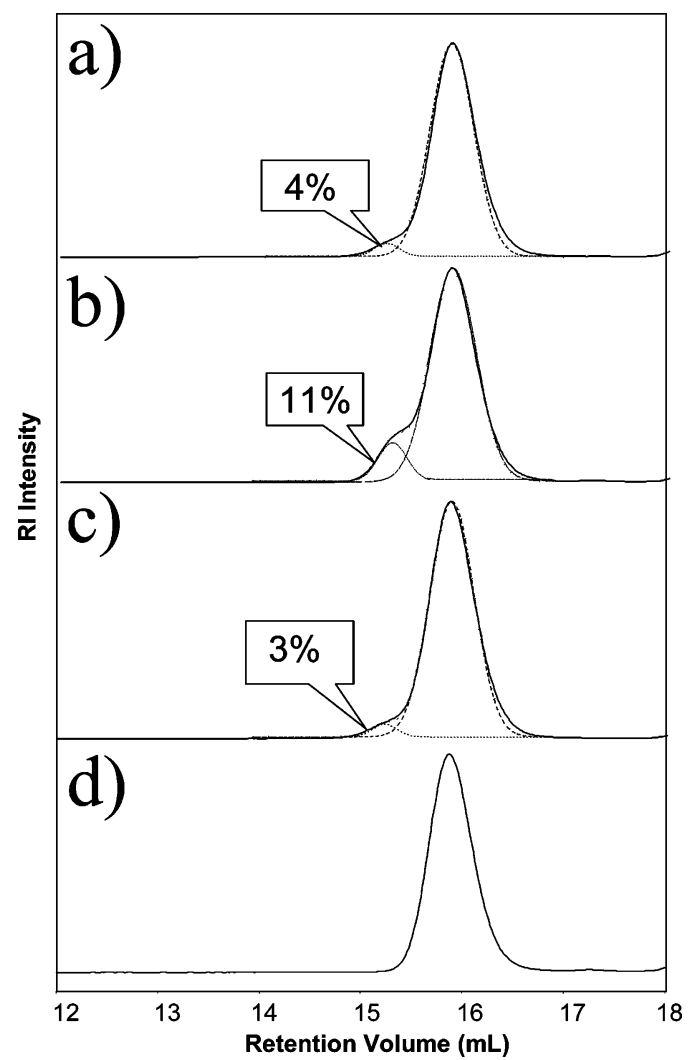

Figure 5. Size exclusion chromatography (SEC) traces of (a) crude product from the reaction of polystyryllithium with 4-bromo-1,1,1-trimethoxybutane, (b) crude product from the reaction of polystyryllithium with succinic anhydride, (c) crude product from the reaction of polystyryllithium with carbon dioxide gas at freeze-dry state, and (d) crude product from the reaction of polystyryllithium with carbon dioxide gas in THF solution at $-78^{\circ} \mathrm{C}$. In all cases, the solid line represents the actual SEC data; the dots are Gaussian components resulting from the fittings.

of the dimerization side reactions in each synthesis. Each curve was approximated by a Gaussian, and the deconvoluted Gaussians are considered as the actual peak. A program in Origin 7.0 was used to conduct these calculations. ${ }^{49}$ In all cases, the solid line is the actual SEC data; the dots are Gaussian components that resulted from the fittings. Two characteristic peaks at retention volumes of 15.3 and $15.9 \mathrm{~mL}$ correspond to dimers and unimers, respectively. The areas of each of these deconvoluted Gaussians are the abundances of these components. The correlation $\left(R^{2}\right)$ value of each fitting is greater than 0.99 . The products from the endcapping reactions of polymeric diphenylalkyllithium give SEC curves identical to chromatogram (d).

Combining the TLC/MALDI-TOF MS and the SEC results, we can see that the reaction of carbon dioxide gas with living polystyryllithium in THF solution at -78 ${ }^{\circ} \mathrm{C}$ produced quantitatively carboxyl-end-capped PS. The product formed from the same reaction but carried out in the freeze-dried state contained $3 \%$ dimers. Note that in the work by Quirk and co-workers ${ }^{9}$ complexation of the active centers with TMEDA was necessary for quantitative carboxylation in the freeze-dried state, and we did not add TMEDA in our work, explaining the presence of some dimers.

\section{Conclusions}

We have demonstrated the use of combined TLC and MALDI-TOF MS to investigate the structures of the 
products from three different anionic strategies for the synthesis of well-defined carboxyl-end-capped polymers. Byproducts that have not been previously detected and reported in the literature were observed by MALDI-TOF MS. The ability to detect byproducts and determine their structures is a prerequisite for gaining a greater understanding of the chain-end-functionalization process. Such information provides synthetic chemists great insight into the reaction efficiency and facilitates the development of synthetic routes that meets the requirements for the application of these materials. An iterative curve-fitting procedure was used to analyze the SEC chromatograms of all crude products. Under the conditions employed, which were in some cases slightly different than those reported previously, only the reaction of carbon dioxide gas and living polystyryllithium in THF solution at $-78{ }^{\circ} \mathrm{C}$ produced carboxyl-endcapped PS quantitatively. The same reaction carried out in the freeze-dried state, even in the absence of Lewis base, was nearly quantitative with only $3 \%$ dimers found via SEC analysis. The reaction of living polystyryl anions with purified succinic anhydride generated about $11 \%$ dimer. Proton-terminated PS was also found to be present in substantial amounts as an additional byproduct. The reaction of living polystyryl anions and purified 4-bromo-1,1,1-trimethoxybutane produced $4 \%$ dimer. In addition to dimers, this reaction also produced a range of byproducts with the following structures: $\mathrm{Bu}-\mathrm{PS}-$ $\mathrm{H}, \mathrm{Bu}-\mathrm{PS}-\mathrm{CH}_{3}, \mathrm{Bu}-\mathrm{PS}-\mathrm{CH}_{2} \mathrm{CH}=\mathrm{CH}-\mathrm{CHO}$, and $\mathrm{Bu}-\mathrm{PS}-\mathrm{CH}_{2} \mathrm{CH}_{2} \mathrm{CH}_{2}-\mathrm{CO}-\mathrm{PS}-\mathrm{Bu}$. The use of DPE to reduce the reactivity of the living anionic chain ends successfully eliminated the formation of dimer in both end-functionalization by succinic anhydride and 4-bromo1,1,1-trimethoxybutane.

Acknowledgment. This work was financially supported by the National Science Foundation through its Collaborative Research in Chemistry Program (CRCCHEM 0304807).

\section{References and Notes}

(1) Hsieh, H. L.; Quirk, R. P. Anionic Polymer, Principles and Practical Application; Marcel Dekker: New York, 1996.

(2) Hirao, A.; Hayashi, M. Acta Polym. 1999, 50, 219-231.

(3) Schulze, J. S.; Cernohous, J. J.; Hirao, A.; Lodge, T. P.; Macosko, C. W. Macromolecules 2000, 33, 1191-1198.

(4) Quirk, R. P.; Ma, J. J. J. Polym. Sci., Part A: Polym. Chem. 1988, 26, 2031-2037.

(5) Quirk, R. P.; Kim, J. Macromolecules 1991, 24, 4515-4522.

(6) Iwasaki, K.; Hirao, A.; Nakahama, S. Macromolecules 1993, $26,2126-2131$.

(7) Charlier, P.; Jerome, R.; Teyssie, P. Macromolecules 1992, $25,617-624$.

(8) Quirk, R. P.; Han, K.; Lee, Y. Polym. Int. 1999, 48, 99-108.

(9) Quirk, R. P.; Yin, J.; Fetters, L. J. Macromolecules 1989, 22, $85-90$.

(10) Hirao, A.; Nakahama, H.; Ishizone, T.; Nakahama, S. Macromolecules 1993, 26, 2145-2150.

(11) Kubo, M.; Mollberg, W. C.; Padis, A. B.; Hall, H. K., Jr.; Calvert, P. Macromolecules 1995, 28, 838-843.

(12) Eastwood, E.; Dadmun, M. D. Macromolecules 2002, 35, 5069-5077.

(13) Eastwood, E.; Dadmun, M. D. Macromolecules 2001, 34, 740747.
(14) Dadmun, M. D. The Compatibilization of Polymer Blends with Linear Copolymers: Comparison Between Simulation and Experiment. In Computational Studies, Nanotechnology, and Solution Thermodynamics of Polymer Systems; Kluwer Academic: New York, 2000.

(15) Eastwood, E.; Dadmun, M. D. Polymer 2002, 43, 6707-6717.

(16) Klein, J. J. Phys.: Condens. Matter 2000, 12, A19-A27.

(17) Grest, G. Adv. Polym. Sci. 1999, 138, 149.

(18) Schorr, P. A.; Kwan, T. C. B.; Kilbey II, S. M.; Shaqfeh, E. S. G.; Tirrell, M. Macromolecules 2003, 36, 389.

(19) DeGennes, P. G. Molecular Conformation and Dynamics of Macromolecules in Condensed Systems; Elsevier: Amsterdam, 1988.

(20) Pasch, H.; Schrepp, W. MALDI-TOF Mass Spectrometry of Synthetic Polymers; Springer-Verlag: Berlin, 2003.

(21) Nielen, M. W. F. Mass Spectrom. Rev. 1999, 18, 309-409.

(22) Arnould, M. A.; Polce, M. J.; Quirk, R. P.; Wesdemiotis, C. Int. J. Mass Spectrom. 2004, 238, 245-255.

(23) Quirk, R. P.; Kim, H.; Polce, M. J.; Wesdemitois, C. Macromolecules 2005, 38, 7895-7906.

(24) Switek, K. A.; Bates, F. S.; Hillmyer, M. A. Macromolecules 2004, 37, 6355-6361.

(25) Hanton, S. D.; Liu, X. M. Anal. Chem. 2000, 72, 4550-4554.

(26) Nielen, M. W. F.; Malucha, S. Rapid Commun. Mass Spectrom. 1997, 11, 1194-1204.

(27) Lee, H.; Chang, T.; Lee, D.; Shim, M.; Ji, H.; Nonidez, W. K.; Mays, J. W. Anal. Chem. 2001, 73, 1726-1732.

(28) Hsieh, S.; Dreisewerd, K.; van der schors, R. c.; Jimenez, C. R.; Stahl-Zeng, J.; Hillenkamp, F.; Jorgenson, J. W.; Geraets, W. M.; Li, K. Anal. Chem. 1998, 70, 1847-1852.

(29) Gusev, A.; Proctor, A.; Rabinovich, Y. I.; Hercules, D. M. Anal. Chem. 1995, 67, 1805-1814.

(30) Hilaire, P. M. St.; Cipolla, L.; Tedebark, U.; Meldal. M. Rapid Commun. Mass Spectrom. 1998, 1475-1484.

(31) Ji, H.; Sato, N.; Nonidez, W. K.; Mays, J. W. Polymer 2002, $43,7119-7123$

(32) Ji, H.; Nonidez, W. K.; Mays, J. W. Int. J. Polym. Anal. Charact. 2002, 7, 181-194

(33) Morton, M.; Fetters, L. J. Rubber Chem. Technol. 1975, 48, 359-409.

(34) Hadjichristidis, N.; Iatrou, H.; Pispas, S.; Pitsikalis, M. J. Polym. Sci., Part A: Polym. Chem. 2000, 38, 3211-3234.

(35) Cornett, D. S.; Duncan, M. A.; Amster, I. J. Anal. Chem. 1993, $65,2608-2613$.

(36) Mowat, I. A.; Donovan, R. J. Rapid Commun. Mass Spectrom. $\mathbf{1 9 9 5}, 9,82-90$.

(37) Karas, M.; Hillenkamp, F. Anal. Chem. 1988, 60, 2299-2301.

(38) Spengler, B.; Pan Y.; Cotter, R. J.; Kan L. S. Rapid Commun. Mass Spectrom. 1990, 4, 99-102.

(39) Montaudo, M. S.; Puglisi, C.; Samperil, F.; Montaudo, G. Rapid Commun. Mass Spectrom. 1998, 12, 519-528.

(40) Ivleva, V. B.; Elkin, Y. N.; Budnik, B. A.; Moyer, S. C.; O'Connor, P. B.; Costello, C. E. Anal. Chem. 2004, 76, 64846491.

(41) Schrimer, D. C.; Li, L. Anal. Chem. 1997, 69, 4176-4183.

(42) Byrd, H. C. M.; McEwen, C. N. Anal. Chem. 2000, 72, 45684576.

(43) Rashidzadeh, H.; Guo, B. Anal. Chem. 1998, 70, 131-135.

(44) Shimada, K.; Lusenkova, M. A.; Sato, K.; Saito, T.; Matsuyama, S.; Nakahara, H.; Kinugasa, S. Rapid Commun. Mass Spectrom. 2001, 15, 277-282.

(45) Guttman, C. M.; Wetzel, S. J.; Flynn, K. M.; Fanconi, B. M.; VanderHart, D. L.; Wallace, W. E. Anal. Chem., in press.

(46) Byrd, H. C. M.; Bencherif, S. A.; Bauer, B. J.; Beers, K. L.; Brun, Y.; Lin-Gibson, S.; Sari, N. Macromolecules 2005, 38, 1564-1572.

(47) Alicata, R.; Montaudo, G.; Puglisi, C.; Samperi, F. Rapid Commun. Mass Spectrom. 2002, 16, 248-260.

(48) Ji, H.; Nonidez, W. K.; Mays, J. W. ANTEC 1998, 2106-2108.

(49) http://www.originlab.com/index.aspx? $=128 \& \operatorname{lm}=63$.

MA050816Q 